\title{
33-fs Yb-fiber laser comb locked to Cs-atomic clock
}

\author{
Ç. Şenel ${ }^{1,2}$, R. Hamid ${ }^{1}$, C. Erdogan ${ }^{1}$, M. Çelik ${ }^{1}$, O. Kara ${ }^{1}$, and F. Ö. Ilday ${ }^{2}$ \\ ${ }^{1}$ TÜBITAK National Metrology Institute (UME), PO 54, TR-41470, Gebze, Kocaeli, Turkey, Turkey \\ ${ }^{2}$ Department of Physics, Bilkent University, 06800 Ankara, Turkey
}

Despite the prevalence of fiber frequency combs around $1.5 \mu \mathrm{m}$, few fully stabilized frequency combs have been demonstrated around $1.0 \mu \mathrm{m}$, despite the generally superior performance of Yb-fiber lasers compared to Er-fiber lasers [1]. Short pulses are to generate coherent supercontinuum using anomalous dispersion regime of microstructured fibers [2]. Near-zero cavity dispersion is highly desirable for low-noise frequency comb performance [3]. Here, we report a Yb-doped fiber laser that operates at net-zero group-velocity dispersion and produces pulses that can be compressed externally to 33-fs. The frequency comb generated by this system is repetitionand carrier-envelope-phase-locked to Cs atomic clocks. The laser oscillator design is based on a novel algorithmic methodology, which allows us to design cavities to meet specific requirements; in this case, there was the need to generate as short pulses as possible, while having several $\mathrm{nJ}$ of pulse energy and the cavity at strictly zero total dispersion.

Experimental setup is shown in Fig. 1(a). Oscillator design relies on self-similar amplification, followed by spectral filtering, then, purely linear dispersive propagation and finally spectral compression in fiber, returning to the original state at the end of the loop. The repetition rate is $49.6 \mathrm{MHz}$. Intracavity pulse energy before (after) the gain fiber is $60 \mathrm{pJ}(4 \mathrm{~nJ})$, limited by pump power. The oscillator was first designed using numerical simulations and then constructed. Extracted pulses are amplified externally in a fiber amplifier that is arranged to exactly mimic pulse propagation inside the oscillator, thereby eliminating gain narrowing. Consequently, the compressed pulse duration after amplification is the same from the oscillator, namely, 33 fs (Fig. 1(b)).
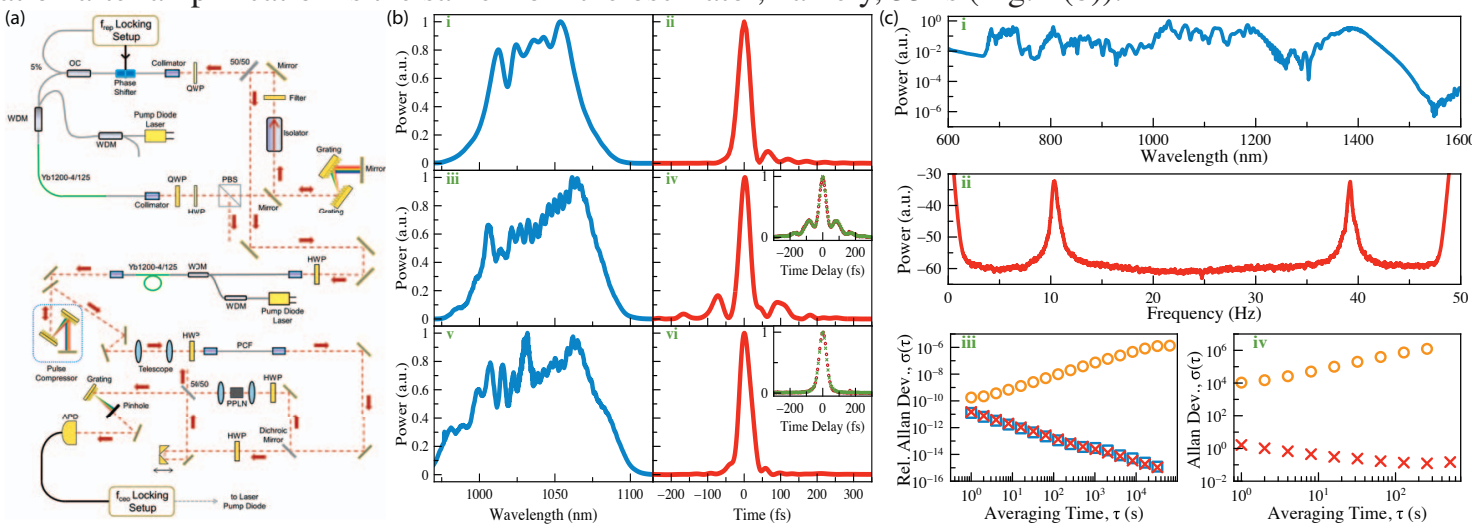

Fig. 1 (a) Schematic diagram of the setup. (b) Optical spectra obtained for (i) simulations of the oscillator, (iii) measured from the oscillator, (v) measured after amplification and retrieved pulse shapes obtained for (ii) the oscillator (simulated), (iv) the oscillator (experimental), (vi) the amplifier (experimental). Insets shows the measured autocorrelations. (c) (i) Spectrum after supercontinuum generation, (ii) free-running $\mathrm{f}_{\text {ceo }}$ beat signals, Allan deviation of (iii) the repetition frequency and (iv) $\mathrm{f}_{\text {ceo }}$ of the free-running fiber comb (circles), Cs-locked fiber comb (squares), and Cs atomic clock (crosses).

Short-term stability of the laser is characterized using relative intensity noise (RIN) and phase noise measurements in the short term and using Allan deviation measurements for frequency stability in the long term (Fig. 1(c)). Integrated RIN from $3 \mathrm{~Hz}$ to $250 \mathrm{kHz}$ is measured as $0.017 \%$. Measured phase noise from $1 \mathrm{kHz}$ to $25 \mathrm{MHz}$ is $76 \mathrm{fs}$ (limited by the measurement setup). After amplification $60 \mathrm{~mW}$ of compressed pulses is coupled to a $30 \mathrm{~cm}$-long segment of photonic crystal fiber (PCF) with zero-dispersion-wavelength of $975 \mathrm{~nm}$ to generate supercontinuum (SC). An f-2f interferometer is built and carrier-envelope-offset beat signals are observed. Repetition and carrierenvelope-offset frequencies $\left(\mathrm{f}_{\mathrm{ceo}}\right)$ are locked to $\mathrm{Cs}$ atomic clocks. The system has been used for absolute frequency measurement of an Nd:YAG/I 2 laser, which is locked to the a10 line of the $\mathrm{R}(56) 32-0$ group.

In conclusion, we report a novel oscillator design, developed using a new theoretical methodology, the output of which was amplified in a novel scheme that eliminates gain filtering and stabilised its repetition rate and $\mathrm{f}_{\text {ceo }}$ to Cs atomic clocks, constituting a fully stabilized frequency comb.

\section{References}

[1] S. A. Diddams, "The evolving optical frequency comb [Invited]," J. Opt. Soc. Am. B 27, B51 (2010).

[2] J. M. Dudley and S. Coen, "Supercontinuum generation in photonic crystal fiber," Rev. Mod. Phys. 78, 1135 (2006).

[3] L. Nugent-Glandorf, T. A. Johnson, Y. Kobayashi, and S. A. Diddams, "Impact of dispersion on amplitude and frequency noise in a Yb-fiber laser comb," Opt Lett 36, 1578 (2011). 